\title{
ESTRATÉGIA PARA MODELAGEM NUMÉRICA DE SISTEMAS MISTOS CONSIDERANDO DIFERENTES MODELOS DE RUPTURA PARA OS MATERIAIS
}

\section{STRATEGY FOR NUMERIC MODELLING OF COMPOSITE SYSTEMS CONSIDERING DIFFERENT MODELS OF RUPTURE FOR THE MATERIALS}

\author{
Julio Cesar Molina ${ }^{1} \quad$ Carlito Calil Junior ${ }^{1}$ \\ Recibido 9 de mayo de 2007, aceptado 04 de junio de 2009 \\ Received: May 9, 2007 Accepted: June 4, 2009
}

\begin{abstract}
RESUMO
Este artigo apresenta a estratégia utilizada na modelagem numérica de um corpo-de-prova misto de madeira e concreto, com sistema de conexão metálico, sob carregamento. A modelagem do sistema foi efetuada a partir de um modelo tridimensional, que utiliza o software ANSYS, que tem como base o Método dos Elementos Finitos, onde foram considerados diferentes modelos de ruptura para os materiais unidos, além de elementos de contato nas interfaces dos mesmos. Os elementos finitos, assim como os materiais utilizados, foram escolhidos em função de suas representatividades perante os comportamentos a serem simulados, para que, dessa forma, fosse possível a consideração de efeitos importantes como plasticidade e ortotropia para os materiais. Os resultados obtidos a partir da modelagem proposta foram comparados com os resultados experimentais de ensaios de cisalhamento, realizados em corpos-de-prova mistos com carregamento aplicado até a ruptura e o erro obtido, neste caso, foi de até $15 \%$. A finalidade deste trabalho é servir de base para a modelagem de sistemas estruturais, presentes em diversas áreas da engenharia, dentre elas, mecânica e civil, onde são utilizados materiais com diferentes comportamentos estruturais. A modelagem proposta possibilita a análise global do modelo assim como a análise dos aspectos localizados de interesse como a concentração de tensões, efeito de difícil quantificação em uma análise experimental.
\end{abstract}

Palabras clave: Sistema misto madeira-concreto, modelagem numérica, ensaios de cisalhamento, modelos de ruptura.

\begin{abstract}
This paper shows the strategy used in the numeric modeling of a composite specimen of wood and concrete, with metallic connection system, with applied cyclical load. The modeling of the system was made starting from a three-dimensional model, using the software ANSYS, that has as base the Method of the Finite Elements, in which different rupture models were considered for the materials, besides contact elements in the interfaces of the materials. The finite elements, as well as the materials used were chosen in function of representativeness for the behaviors to be simulated, in thus was possible the consideration of important effects as plasticity and ortotropia for the materials. The results obtained from the proposed modeling were compared with the experimental results of shear tests in composite specimens with load applied until the rupture, and the error obtained, in this case, was $15 \%$. The aim of this work is to serve as base for the modeling of structural systems, present in several areas of engineering, among them, mechanics and civil, where materials with different structural behaviors are used. The proposed modeling makes possible the global analysis of the model and locates aspects of interest as the concentration of stress, effect of difficult quantification in an experimental analysis.
\end{abstract}

Keywords: Wood-concrete composite systems, numeric modeling, push-out tests, rupture models.

\section{INTRODUÇÃO}

A grande maioria dos modelos numéricos analisados no âmbito das engenharias, onde se utiliza a análise computacional, para modelos numéricos, propõe formas simplificadas de análise sem considerar os critérios de resistência, ruptura ou falha dos materiais. Os modelos procuram, de uma maneira geral, restringir o comportamento das estruturas a limites linearmente elásticos ao invés de considerar os verdadeiros critérios de ruptura para os materiais obtendo, dessa forma, respostas muito conservadoras. Pode-se dizer que as

1 EESC/USP. Av. Trabalhador São Carlense, 400, Centro, São Carlos-SP. CEP 13560-970. Brasil. E-mail: juliocesarmolina@ yahoo.com.br 
estruturas assim analisadas, são capazes de resistir a esforços significativamente superiores aos esforços de projeto. Porém, as tensões e deformações geradas pelas solicitações externas frequentemente excedem os limites elásticos dos materiais e, portanto, a resposta estrutural dos materiais não deve ser estudada como linearmente elástica. Os resultados obtidos por uma análise linear são válidos à medida que as deformações plásticas são pequenas e, por outro lado, para solicitações com valores últimos, tem-se a falha dos materiais. Além disso, a utilização de modelos numéricos que envolvam dois ou mais materiais estruturais consiste, quase sempre, em uma tarefa relativamente trabalhosa.

Investigações anteriores realizadas por [1-2] mostram que os resultados das simulações numéricas tiveram proximidade com os resultados dos ensaios experimentais, para baixos valores de carregamentos aplicados. De acordo com [3-4], os resultados fornecidos pela simulação numérica foram distantes daqueles observados em ensaios de laboratório e, segundo os autores, estes resultados seriam melhorados com a utilização de um modelo capaz de representar os efeitos da não linearidade física dos materiais utilizados.

Ainda neste contexto, sabe-se que a geometria dos elementos estruturais, especialmente nas regiões próximas as conexões são capazes de elevar os estados de tensões a níveis máximos atingindo a falha dos materiais para carregamentos de serviço. Por estas razões, é de fundamental importância, em uma análise estrutural, independentemente do material utilizado, a utilização de modelos mais sofisticados que permitam determinar com maior precisão o verdadeiro comportamento estrutural dos materiais, principalmente nas regiões das conexões dos materiais. Assim, para carregamentos de serviço, sempre que possível, os modelos devem considerar além do comportamento não-linear nos trechos inelásticos, a plastificação dos materiais, e também os efeitos oriundos da ortotropia dos materiais, quando necessários, procurando, deste modo, evitar possíveis deficiências dos materiais em condições de projeto (serviço).

No entanto, atualmente, a análise numérica tem avançado bastante no sentido de descrever os fenômenos reais das estruturas. Uma das ferramentas utilizadas, neste caso, é o Método dos Elementos Finitos (MEF), associado a consideração de modelos de ruptura para os materiais utilizados na composição dos sistemas estruturais, principalmente nos sistemas estruturais mistos.

Os resultados das simulações numéricas desenvolvidas por [5-8] onde foram utilizados diferentes modelos de ruptura para os materiais, mostram boas correlações com os resultados experimentais para as curvas carga versus deslizamento.

Entre os modelos de ruptura pode-se citar o modelo de Hill [9], freqüentemente utilizado para descrever o comportamento de materiais ortotrópicos, e o modelo de von Mises [10], utilizado para descrever o comportamento de grande parte dos materiais isotrópicos, entre outros modelos.

O modelo de Hill considera que a ruptura do material independe da pressão hidrostática. Esse modelo considera diferentes tensões de plastificação nas direções principais do material e um fluxo de tensão associado. O modelo de ruptura de von Mises, também conhecido como critério da máxima energia de distorção, é baseado em conceitos de energia de deformação. Neste modelo a energia elástica total do sistema é dividida em duas partes: uma associada a mudanças volumétricas do material e a outra causando distorções por cisalhamento. Igualando-se a energia de cisalhamento no ponto de escoamento à tração simples, com aquela sob tensão combinada, é estabelecido o critério de escoamento para a tensão combinada.

Neste cenário se insere o presente trabalho de pesquisa trazendo contribuições, principalmente, no que se refere a análise numérica de sistemas estruturais mistos, ou seja, sistemas estruturas onde são utilizados diferentes materiais, a partir da utilização do software ANSYS [11] e também de elementos finitos sólidos.

Neste artigo, apresenta-se, portanto, uma estratégia de modelagem numérica, com a finalidade de estimular a utilização de algumas ferramentas disponibilizadas pela biblioteca interna do ANSYS [11], para a análise de diferentes sistemas estruturais, presentes nas áreas da engenharia, que envolvem a utilização de modelos numéricos na análise estrutural. Assim, independentemente do material considerado, são aqui apresentados os principais parâmetros (elásticos e plásticos) utilizados pelo ANSYS na modelagem de sistemas estruturais que necessitem da utilização de modelos de ruptura para caracterização de materiais com comportamento isotrópico, não linearidade física e também com ortotropia nas soluções estruturais.

Para exemplificar, propõe-se a análise de um modelo numérico, tridimensional, de corpo-de-prova misto, que utiliza os materiais madeira e concreto, com sistema de conexão metálico. Este modelo foi submetido a um carregamento estático, aplicado até o valor de ruptura, conforme obtido experimentalmente, para a calibração do sistema, sendo que a modelagem do sistema misto foi 
efetuada, neste caso, a partir da utilização do software ANSYS, versão 9.0, que tem como base o Método dos Elementos Finitos (MEF). A opção pela utilização do ANSYS se deu pelo fato de que as ferramentas disponibilizadas pelo referido software permitem a análise dos modelos em regime de não linearidade física e geométrica, além da permitir a utilização de elementos de contato entre os materiais.

O modelo de corpo-de-prova misto, proposto no exemplo, frequentemente é utilizado na verificação do comportamento de conectores de cisalhamento, utilizados na união de diferentes materiais, em diversos sistemas estruturais mistos.

Para tanto, na seqüência estão apresentados os aspectos gerais da estratégia de modelagem desenvolvida, juntamente com os comentários mais relevantes.

\section{ASPECTOS GERAIS DA ESTRATÉGIA DE MODELAGEM NUMÉRICA}

Configuração do modelo: $\mathrm{O}$ modelo de corpo-deprova analisado, e tomado como exemplo neste artigo, foi composto por duas peças laterais de concreto, com resistência média na compressão de $28 \mathrm{MPa}$, e por uma peça central de madeira, da espécie Eucalipto citriodora, de classe de resistência C60. Segundo [12], as espécies de madeira que se incluem na classe C60 apresentam o valor médio da resistência a compressão, na direção das fibras, igual a $60 \mathrm{MPa}$. Os valores das propriedades mecânicas da madeira utilizada no modelo numérico, tomado como exemplo, estão apresentados na tabela 4 . $\mathrm{O}$ valor da resistência a compressão tomada na direção das fibras, neste caso, foi igual a 62,80 MPa. Para a união dos referidos materiais foram utilizados um total de dois conectores de cisalhamento, dispostos em " $\mathrm{X}$ " (fixados com inclinação de $45^{\circ}$ em relação às fibras da madeira), formados por barras de aço, do tipo CA-50, com $8 \mathrm{~mm}$ de diâmetro, coladas nas peças de madeira com resina epóxi Sikadur 32. Os comprimentos de ancoragem na madeira e no concreto foram respectivamente $8 \mathrm{~cm}$ e $20 \mathrm{~cm}$. Para diminuição do tempo de processamento do modelo, optouse ainda pela modelagem de $1 / 2$ corpo-de-prova.

Elemento finito Solid65: Foi utilizado na discretização das peças de concreto. A opção por este elemento se deu pelo fato da possibilidade de simulação dos efeitos localizados como, por exemplo, a concentração de tensões junto aos conectores de cisalhamento. O elemento solid65 é um elemento hexaédrico, que possui oito nós, tendo cada nó três graus de liberdade, ou seja, translações segundo os eixos $\mathrm{x}, \mathrm{y}$ e z. Este elemento é capaz de simular o comportamento do concreto com fissuração na tração e esmagamento na compressão, bem como um comportamento com não-linearidade física, o que permite avaliar as deformações plásticas. Além disso, as armaduras podem ser incluídas sob a forma de taxas, denominadas armaduras dispersas, orientadas, neste caso, segundo os ângulos $\varphi$ e $\theta$, ou ainda, pela utilização de elementos barra na forma discreta, procedimento este adotado para o modelo numérico do presente artigo.
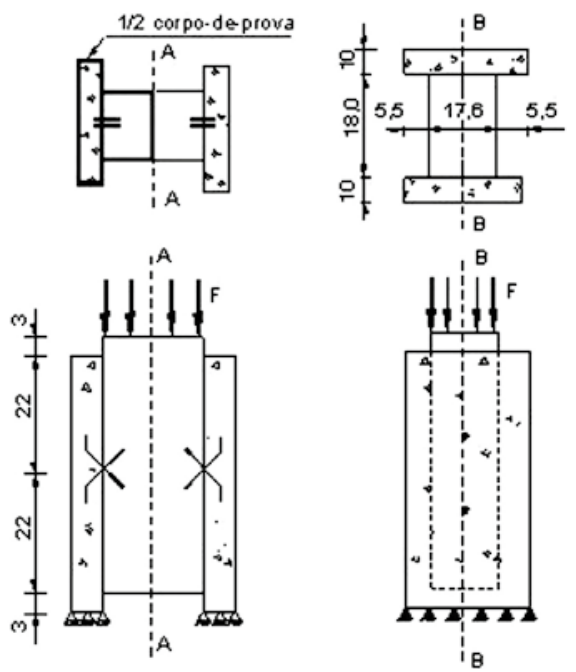

Figura 1. Modelo completo de corpo-de-prova (medidas em $\mathrm{cm}$ ). Fonte: Adaptado de [8].

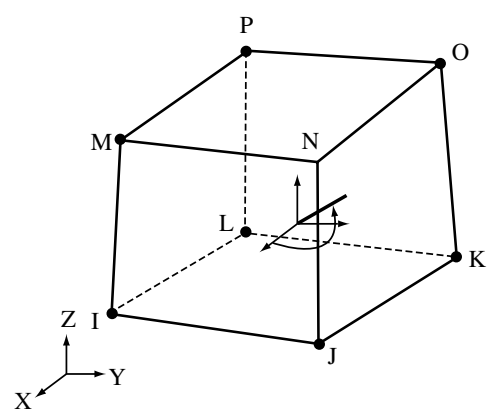

Figura 2. Elemento finito solid65. Fonte: Adaptado da documentação [11].

Elemento finito Solid45: Foi utilizado na discretização das peças de madeira e também dos conectores de cisalhamento. O elemento solid45 também consiste num elemento hexaédrico, com oito nós, tendo cada nó três graus de liberdade (translações segundo os eixos $\mathrm{x}, \mathrm{y}$ e $\mathrm{z}$ ), e permite ainda a consideração de efeitos importantes como, por exemplo, plasticidade e ortotropia para os materiais. 


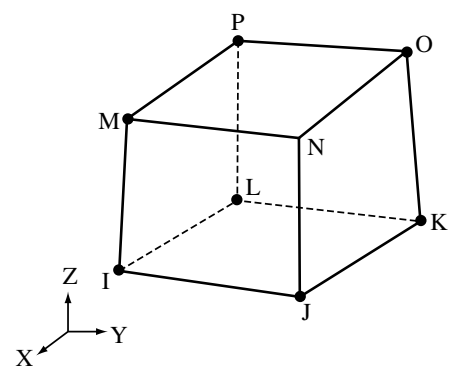

Figura 3. Elemento finito solid45. Fonte: Adaptado da documentação [11].

Elemento finito link8: Foi utilizado na discretização das armaduras de aço imersas no concreto. O elemento link8 consiste num elemento tridimensional de barra, que possui dois nós, cada nó com três graus de liberdade (translações segundo os eixos x, y e z), e responde a esforços de tração e compressão axiais. O eixo " $x$ " do elemento é orientado segundo o seu comprimento e nenhuma flexão no elemento é considerada. Porém, é possível admitir a ocorrência de deformação plástica para o material.

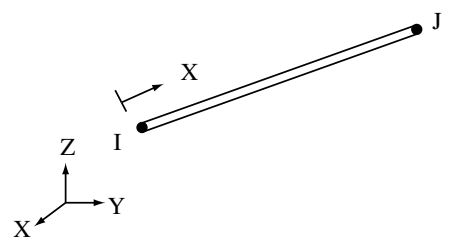

Figura 4. Elemento finito link8. Fonte: Adaptado da documentação [11].

Elementos conta173 e targe170: Estes elementos foram utilizados a fim de representar os contatos existentes com possíveis deslocamentos nas seguintes interfaces: madeira-concreto, aço-concreto e aço-madeira, pois a ligação por pinos é do tipo semi-rígida. Esses elementos são utilizados em análises tridimensionais, com contato do tipo superfície-superfície, que surge do trabalho em conjunto dos elementos targe 170 (definido pelo ANSYS como superfície alvo) e conta173 (definido como superfície de contato). Esses elementos são capazes de simular a existência de pressão entre os elementos, quando há contato, e separação entre os mesmos elementos quando não há contato. Os pares de contato utilizados permitem ainda a consideração do atrito entre as partes. Os valores dos coeficientes de atrito utilizados entre os pares de contato aço-concreto e aço-madeira foram admitidos com base em [6], ou seja, aço-concreto $=0,60$ e aço-madeira $=0,50$. $\mathrm{O}$ valor do coeficiente de atrito utilizado na interface madeira-concreto foi de 0,01 , tendo-se em vista que durante a realização dos ensaios experimentais de cisalhamento foram utilizados sacos plásticos de polietileno na referida interface dos materiais visando a eliminação do atrito entre os mesmos. Esses valores para os coeficientes de atrito foram os que possibilitaram as melhores respostas com relação ao comportamento simulado.

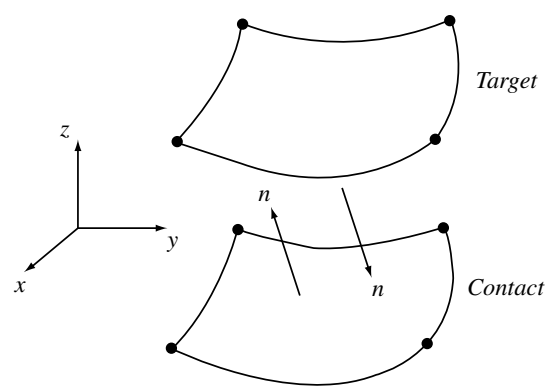

Figura 5. Elementos de contato. Fonte: Adaptado da documentação [11].

Determinação da malha de elementos finitos: $\mathrm{O}$ modelo desenvolvido foi constituído por três principais grupos de elementos: peças de concreto, peça de madeira e conectores de cisalhamento. Nos elementos referentes às peças de concreto foram incluídas as armaduras. Um quarto grupo de elementos foi constituído pelos elementos de contato na interface entre os materiais. Todos os elementos utilizados foram discretizados separadamente, porém de forma a coincidir os nós de cada um na interface entre eles, possibilitando o acoplamento entre tais elementos, realizado por meio destes nós. As malhas dos elementos foram geradas e discretizadas no software TrueGrid [13], versão 2.10 , sendo posteriormente exportadas para o ANSYS, onde receberam as correspondentes propriedades dos materiais, condições de vinculação e carregamentos, necessárias para a análise do sistema misto em questão. O TrueGrid foi uma ferramenta fundamental na geração das malhas dos elementos, principalmente devido a necessidade de obtenção de um maior grau de refinamento nas regiões das conexões. Tendo-se em vista a dificuldade de construção e de análise do modelo numérico, foram criados scripts, contendo as seqüências de comandos, os quais foram executados pelo ANSYS, permitindo-se assim a geração e análise automática dos modelos. Os comandos necessários para a análise do modelo numérico no ANSYS foram escritos em função das propriedades elásticas e plásticas dos materiais, do comportamento da curva tensão versus deformação experimental, entre outros. A conversão da linguagem de programação do através da utilização do recurso do programa TGEdit que é um editor próprio do TrueGrid [13].

Discretização da malha de elementos finitos: A malha do modelo numérico foi analisada a partir de diferentes níveis de refinamento, até que os resultados conduzissem a respostas satisfatórias em termos de deslocamentos. Nas regiões das conexões as malhas foram mais discretizadas. 
Na Figura 6, os parâmetros relacionados em (a) são referentes à peça de concreto, em (b) às peça de madeira, em (c) e (d) às peça de madeira como também de concreto, e em (e) à discretização dos conectores de aço dispostos em "X".

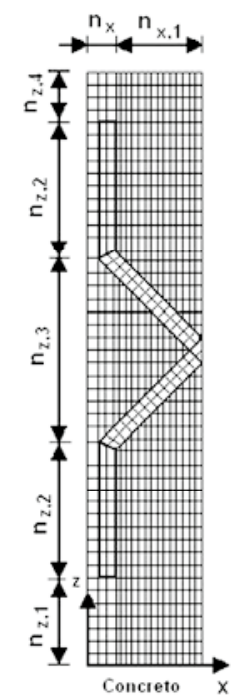

(a)

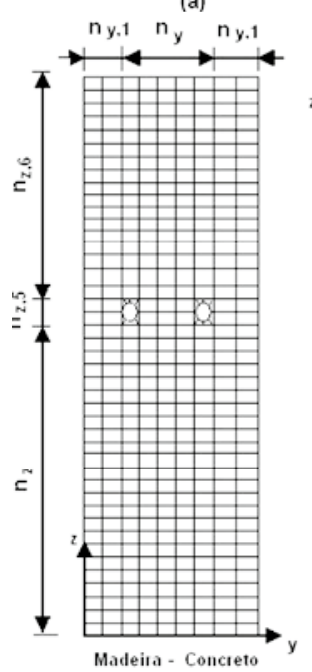

(c)

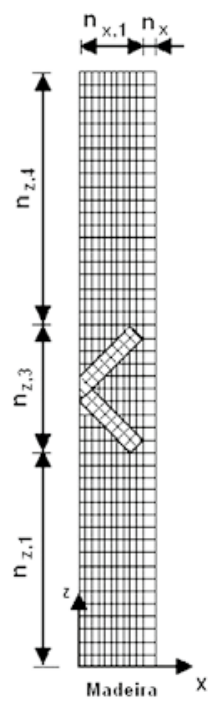

(b)

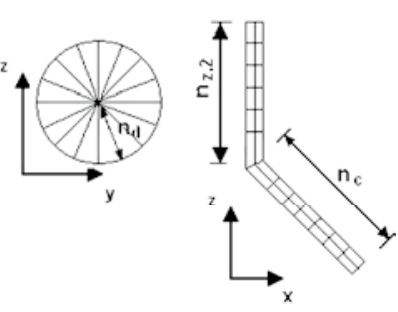

Conector de aço

(e)

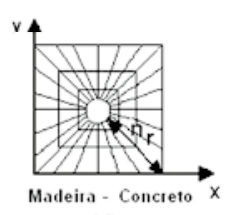

(d)
Figura 6. Parâmetros utilizados na definição da malha de elementos finitos, considerando $1 / 2$ estrutura. Fonte: Adaptado de [8].

onde:

$\mathrm{n}_{\mathrm{x}}=$ número de elementos de extremidade na direção da espessura do elemento (concreto ou madeira);

$\mathrm{n}_{\mathrm{x}, 1}=$ número de elementos na direção da espessura do elemento (concreto ou madeira);

$\mathrm{n}_{\mathrm{y}}=$ número de elementos centrais na direção da largura do elemento (concreto ou madeira);

$\mathrm{n}_{\mathrm{y}, 1}=$ número de elementos nas extremidades na direção da largura do elemento (concreto ou madeira); $\mathrm{n}_{\mathrm{z}}=$ número de elementos na extremidade inferior, abaixo da linha de conectores, na direção da altura do elemento (madeira ou concreto);

$\mathrm{n}_{\mathrm{z}, 1}=$ número de elementos na extremidade inferior, na direção da altura do elemento (madeira ou de concreto);

$\mathrm{n}_{\mathrm{z}, 2}=$ número de elementos na região vertical do conector na direção da altura do elemento (concreto);

$\mathrm{n}_{\mathrm{z}, 3}=$ número de elementos centrais na direção da altura do elemento (madeira ou concreto);

$\mathrm{n}_{\mathrm{z}, 4}=$ número de elementos na extremidade superior na direção da largura do elemento (concreto ou madeira);

$\mathrm{n}_{\mathrm{z}, 5}=$ número de elementos centrais, na linha de conectores, na direção da altura do elemento (concreto ou de madeira);

$\mathrm{n}_{\mathrm{z}, 6}=$ número de elementos na extremidade superior, acima da linha de conectores, na direção da altura do elemento (madeira ou concreto);

$\mathrm{n}_{\mathrm{c}}=$ número de elementos, na direção inclinada do conector, considerando os trechos de madeira e também de concreto;

$\mathrm{n}_{\mathrm{r}}=$ número de elementos na direção do raio dos conectores (madeira ou concreto);

$\mathrm{n}_{\mathrm{d}}=$ número de elementos na direção da espessura do conector.
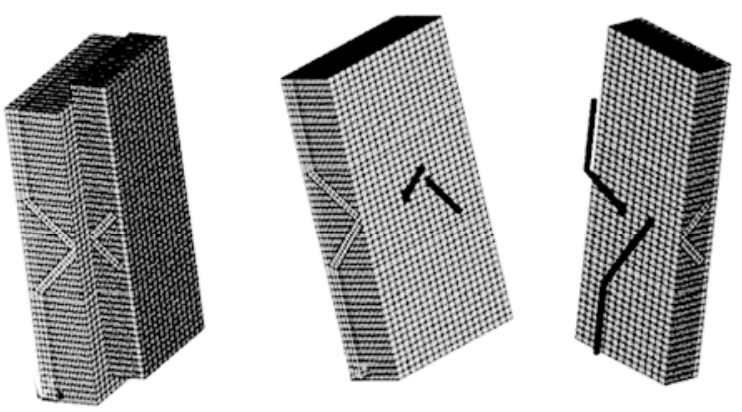

a) Corpo-de-prova misto b) Detalhe dos conectores

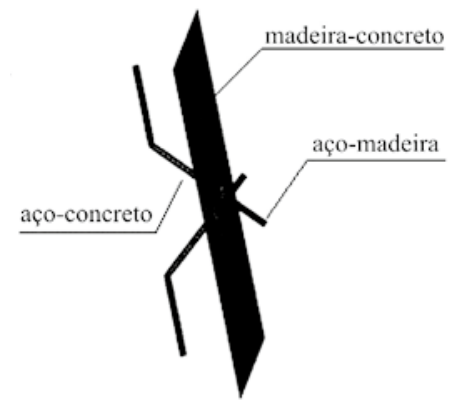

c) Elementos de contato

Figura 7. Detalhe do modelo considerando $1 / 2$ estrutura. Fonte Adaptado de [8]. 
Modelo de ruptura para o concreto: $O$ comportamento do concreto na tração foi representado pelo modelo concrete, disponibilizado na biblioteca do ANSYS, que tem como base o modelo de Willan-Warnke, através do qual é possível a simulação da fissuração do concreto quando submetido a tensões de tração. Os parâmetros necessários para a utilização deste modelo foram:

- coeficiente de transferência de cisalhamento para a fissura aberta;

- coeficiente de transferência de cisalhamento para a fissura fechada;

- resistência última uniaxial à tração $\left(f_{t}\right)$;

- resistência última uniaxial à compressão $\left(\mathrm{f}_{\mathrm{c}}\right)$;

- resistência última biaxial à compressão $\left(\mathrm{f}_{\mathrm{cb}}\right)$;

- estado de tensão hidrostática ambiente $\left(\sigma_{\mathrm{ha}}\right)$;

- resistência última biaxial à compressão sob o estado de tensão hidrostático $\left(\mathrm{f}_{1}\right)$;

- resistência última uniaxial à compressão sob o estado de tensão hidrostático $\left(\mathrm{f}_{2}\right)$;

- coeficiente multiplicador de rigidez para condição fissurada na tração.

Com base no trabalho [14], foram adotados para os dois primeiros parâmetros do modelo, os valores 0,2 e 0,6, respectivamente. Para o terceiro parâmetro foi adotado o valor de $10 \%$ da resistência do concreto na compressão. Os últimos cinco parâmetros foram omitidos, permitindo-se ao ANSYS admitir valores pré-estabelecidos para os mesmos. A resposta do concreto na compressão, disponibilizada pelo modelo concrete, foi desabilitada estipulando-se para tanto, neste modelo, $\mathrm{f}_{\mathrm{c}}$ igual a-1. Admitiu-se, assim, o critério de plastificação de von Mises para o concreto na compressão. $\mathrm{O}$ modelo constitutivo adotado para o concreto na compressão foi do tipo multilinear com encruamento isótropo.

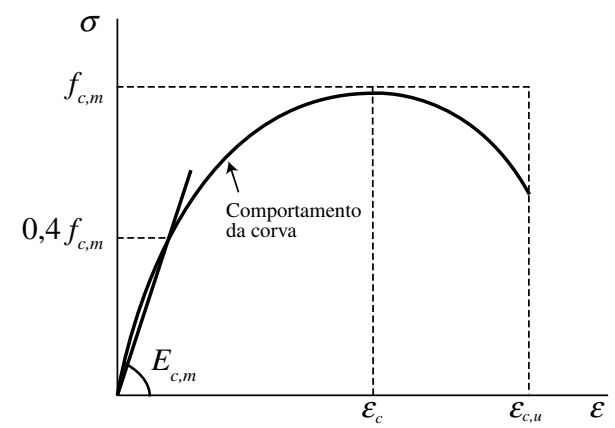

Figura 8. Modelo de ruptura adotado para o concreto. Fonte: Adaptado de [8].

$\mathrm{Na}$ figura anterior $\mathrm{f}_{\mathrm{cm}}$ é o valor médio de resistência à compressão do concreto para corpo-de-prova cilíndrico, $\varepsilon_{\mathrm{c}}$ a deformação correspondente à tensão máxima de compressão, $\varepsilon_{\mathrm{c}, \mathrm{u}}$ a deformação última na compressão e, $\mathrm{E}_{\mathrm{c}, \mathrm{m}}$ o módulo secante de elasticidade do concreto. A curva tensão-deformação utilizada, neste caso, foi obtida a partir de ensaios experimentais, realizados em corpos-de-prova cilíndricos de concreto, com dimensões de $10 \mathrm{~cm}$ x $20 \mathrm{~cm}$.

Modelo de ruptura para a madeira: Na primeira tentativa de modelagem da madeira, admitiu-se para o material um comportamento isotrópico (mesmas propriedades físicas independentemente da direção considerada), com critério de resistência de von Mises, associado ao encruamento isótropo. Porém, os resultados obtidos para a curva Força versus Deslocamento, neste caso, não foram satisfatórios. Optou-se então pela consideração de um comportamento ortotrópico (diferentes propriedades físicas para cada uma das três direções consideradas) para a madeira, com a utilização do critério de resistência de resistência de Hill, associado ao encruamento isótropo. O critério de Hill [9] é uma extensão do critério de von Mises (critério da máxima energia de distorção), para consideração da anisotropia dos materiais, onde a ruptura do material independe das tensões hidrostáticas e considera, neste caso, uma regra de fluxo associada, um endurecimento de trabalho, junto com diferentes tensões de plastificação nas três direções principais do material.

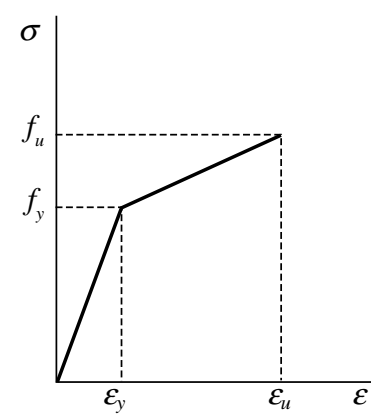

Figura 9. Modelo de ruptura adotado para a madeira. Fonte: Adaptado de [8].

A modelagem da madeira exigiu a utilização de um total de 21 constantes, sendo estas constantes referentes às propriedades elásticas e plásticas da madeira. Por simplicidade, os comportamentos da madeira na tração e na compressão foram considerados iguais. Além disso, nenhuma distinção foi feita entre as direções radial e tangencial (isotropia transversal) e os valores considerados, neste caso, corresponderam à direção perpendicular às fibras da madeira (direção radial). As propriedades elásticas da madeira, $E_{z}$ e $G$, conforme apresentadas na seqüência, foram obtidas através de ensaios experimentais realizados em corpos-de-prova de madeira, segundo recomendações da [12]. As demais propriedades elásticas e as relações entre elas foram admitidas com base nas recomendações de [15]. Os valores das propriedades plásticas da madeira, assim como as relações entre eles, foram tomados com 
base nos trabalhos [5] e [6]. Admitiu-se, portanto, para o valor da tensão última de plastificação da madeira $\left(\sigma_{\mathrm{z}}\right)$, na direção das fibras, o mesmo valor da resistência da madeira na compressão, ou seja, 62,80 MPa. No ANSYS, o eixo de coordenadas "z" correspondeu à direção longitudinal das fibras da madeira, o eixo "y" à direção radial, e o eixo "x" à direção tangencial.

O ensaio experimental de cisalhamento do corpo-de-prova misto, para calibração do modelo numérico, foi realizado com aplicação de três ciclos de carga, sendo os dois primeiros ciclos com carregamento aplicado até $50 \%$ da resistência da ligação (determinada a partir de um corpo-de-prova gêmeo e correspondente a deformação $2 \%$ o e o terceiro ciclo com carregamento aplicado até a ruptura, conforme indicações da [12]. Os carregamentos aplicados, neste caso, foram controlados por uma célula de carga com capacidade de $250 \mathrm{kN}$ e o sistema de solicitação do corpo-de-prova foi desenvolvido por meio de um pórtico de reação. Os deslocamentos entre os materiais madeira e concreto foram medidos através de dois transdutores de deslocamento com sensibilidade de $0,001 \mathrm{~mm}$ e percurso máximo de 5 $\mathrm{mm}$, colocados em ambos os lados do corpo-de-prova. A base de medida considerada para o corpo-de-prova foi de $33 \mathrm{~cm}$. Foi utilizado também um sistema de aquisição de dados externo como um total de 20 canais sendo dois deles para os transdutores de deslocamento e um para a célula de carga. A carga última aplicada no corpo-de-prova, durante o ensaio, foi de $61,16 \mathrm{kN}$.

As constantes utilizadas para a modelagem numérica da madeira, assim como as relações utilizadas entre elas, e que foram obtidas a partir de [5], [6], [12] e [15], estão apresentadas na seqüência.

\section{Propriedades elásticas da madeira:}

$\mathrm{E}_{\mathrm{x}} \pm=$ módulo de elasticidade na direção tangencial;

$\mathrm{E}_{\mathrm{y}} \pm=$ módulo de elasticidade na direção radial;

$\mathrm{E}_{\mathrm{z}}{ }^{ \pm}=$módulo de elasticidade na direção longitudinal (compressão);

$v_{\mathrm{xy}}=$ coeficiente de Poison no plano xy;

$v_{\mathrm{yz}}=$ coeficiente de Poison no plano yz;

$v_{\mathrm{xz}}=$ coeficiente de Poison no plano xz;

$\mathrm{G}_{\mathrm{xy}}=$ módulo de elasticidade transversal no plano $\mathrm{xy}$;

$\mathrm{G}_{\mathrm{yz}}=$ módulo de elasticidade transversal no plano yz;

$\mathrm{G}_{\mathrm{xz}}=$ módulo de elasticidade transversal no plano $\mathrm{xz}$;

\section{Propriedades plásticas da madeira:}

$\sigma_{\mathrm{x}} \pm=$ tensão de plastificação (tração e compressão) na direção $\mathrm{x}$;

$\sigma_{\mathrm{y}} \pm=$ tensão de plastificação (tração e compressão) na direção y;

$\sigma_{\mathrm{z}^{ \pm}}=$tensão de plastificação (tração e compressão) na direção z;
$\mathrm{E}_{\mathrm{Tx}} \pm=$ módulo tangente (tração e compressão) na direção $\mathrm{x}$;

$\mathrm{E}_{\mathrm{Ty}} \pm=$ módulo tangente (tração e compressão) na direção $\mathrm{y}$

$\mathrm{E}_{\mathrm{Tz}^{ \pm}} \pm=$módulo tangente (tração e compressão) na direção $\mathrm{z}$

$\tau_{\mathrm{xy}}=$ tensão cisalhante de plastificação na direção xy;

$\tau_{\mathrm{yz}}=$ tensão cisalhante de plastificação na direção yz;

$\tau_{\mathrm{xz}}=$ tensão cisalhante de plastificação na direção xz;

$\mathrm{G}_{\mathrm{Txy}}=$ módulo tangente de corte no plano xy;

$\mathrm{G}_{\mathrm{Tyz}}=$ módulo tangente de corte no plano yz;

$\mathrm{G}_{\mathrm{Txz}}=$ módulo tangente de corte no plano $\mathrm{xz}$;

Relações admitidas entre as propriedades elásticas da madeira:

$\mathrm{E}_{\mathrm{x}}=\mathrm{E}_{\mathrm{y}}=\left(\mathrm{E}_{\mathrm{z}} / 10\right)$;

$\mathrm{G}=\mathrm{G}_{\mathrm{xy}}^{\mathrm{y}}=\mathrm{G}_{\mathrm{yz}}=\mathrm{G}_{\mathrm{xz}}=\left(\mathrm{E}_{\mathrm{z}} / 20\right)$;

$v_{\mathrm{xy}}=0,23 ; v_{\mathrm{yz}}=v_{\mathrm{xz}}=0,013$

$\mathrm{G}_{\mathrm{Txy}}=\mathrm{G}_{\mathrm{Tyz}}=18 ; \mathrm{G}_{\mathrm{Txz}}=0,018$

Relações admitidas entre as propriedades plásticas da madeira:

$\sigma_{\mathrm{z}}=\mathrm{f}_{\mathrm{c} 0, \mathrm{~m}}=\mathrm{f}_{\mathrm{u}}$

$\left(\sigma_{\mathrm{y}} / \sigma_{\mathrm{z}}\right)=\left(\sigma_{\mathrm{x}} / \sigma_{\mathrm{z}}\right)=0,19$

$\tau_{\mathrm{xy}}=\tau_{\mathrm{yz}}=0,38 \sigma_{\mathrm{z}} ; \tau_{\mathrm{xz}}=0,038 \sigma_{\mathrm{z}}$

$\mathrm{E}_{\mathrm{Tx}}=\mathrm{E}_{\mathrm{Ty}}=4,1 ; \mathrm{E}_{\mathrm{Tz}}=280$

Modelo de ruptura para o aço dos conectores: Para os conectores de cisalhamento foi adotado um modelo bilinear, com encruamento isótropo, e critério de plastificação de von Mises. A caracterização do comportamento isotrópico admitido para do aço exigiu a utilização de quatro constantes: $\mathrm{E}$ (Módulo de elasticidade), $\sigma_{\mathrm{p}}$ (tensão de plastificação), $\mathrm{E}_{\mathrm{T}}$ (módulo tangente) e $v$ (coeficiente de Poison). Os valores das referidas constantes, neste caso, foram os mesmo utilizados por [5].

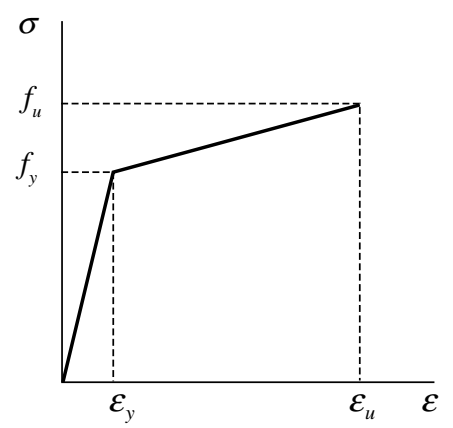

Figura 10. Modelo de ruptura adotado para o aço dos conectores. Fonte: Adaptado de [8].

Modelo de ruptura para 0 aço das armaduras: A relação constitutiva utilizada para o aço da armadura também seguiu o critério de von Mises, sendo representada, neste caso, por meio da curva tensão versus deformação, com base 
em um modelo elasto-plástico perfeito. A fim de evitar problemas numéricos no trecho da curva que vai de $\varepsilon_{\mathrm{y}}$ a $\varepsilon_{\mathrm{u}}$ foi considerada uma pequena inclinação de E/1000.

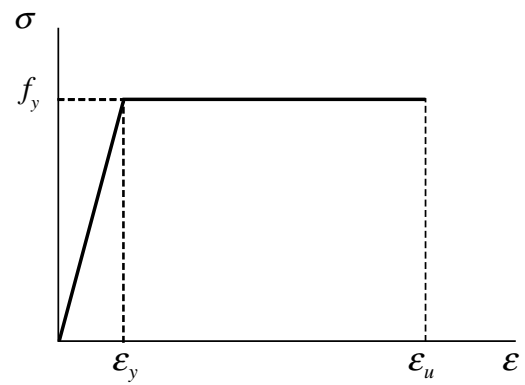

Figura 11. Modelo constitutivo adotado para o aço da armadura. Fonte: Adaptado de [8].

Condições de contorno, vinculação e carregamento: Para garantir a estabilidade do modelo durante a aplicação do carregamento foram respeitadas as condições de simetria, e também de vinculação dos nós dos apoios. O modelo analisado foi vinculado em concordância com o ensaio experimental. Vale lembrar que os nós dos elementos utilizados nos modelos apresentam apenas três graus de liberdade que são referentes às translações em x, y e z (coordenadas locais).

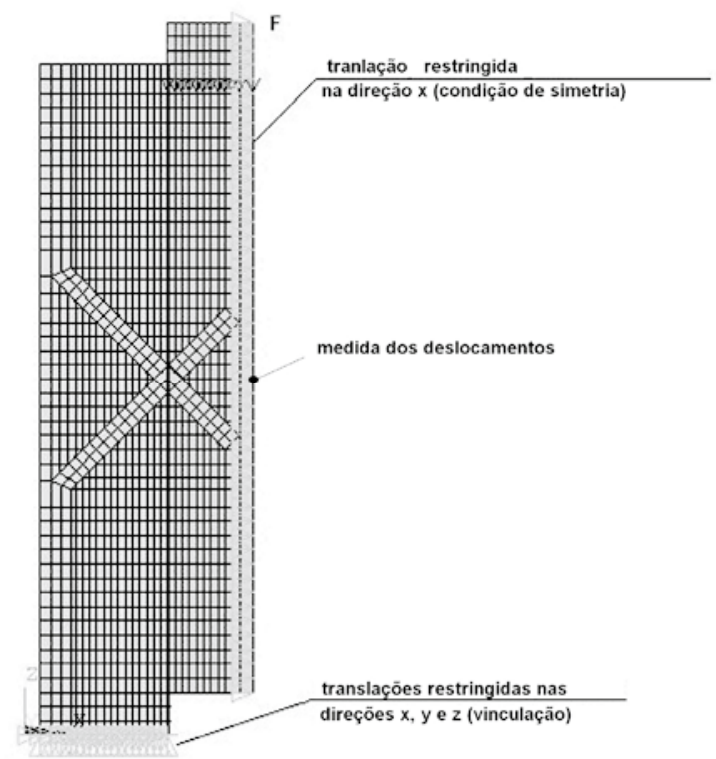

Figura 12. Vinculação e condições de simetria para o corpo-de-prova. Fonte: Adaptado de [8].

A validação do modelo foi efetuada a partir da comparação do comportamento das curvas Força versus Deslocamento (numérico-experimental). A intensidade de força aplicada foi definida por meio da divisão da força de ruptura estimada na análise experimental (considerando $1 / 2$ de estrutura) pelo número de nós presentes na região relativa a aplicação da força. Na base superior do corpo-de-prova foi aplicada uma força $F=61,16 \mathrm{kN}$, definida com base no ensaio experimental do corpo-de-prova, até a ruptura do sistema, para a avaliação da curva força versus deslizamento (calibração do modelo).

Aspectos da análise não linear: $O$ carregamento no modelo analisado foi aplicado de forma incremental, devido à consideração da não linearidade física. $\mathrm{O}$ incremento de carga foi controlado utilizando-se o recurso do ANSYS denominado "Automatic Time Stepping". De acordo com a documentação do ANSYS, o recurso citado reduz o valor do incremento quando a previsão do número de iterações ultrapassa o limite estabelecido, caso se obtenha incrementos de deformações plásticas maiores que $15 \%$, ou ainda, deslocamentos excessivos. $\mathrm{O}$ valor do incremento pode também ser aumentado, caso o processo venha a convergir de forma sistemática na primeira iteração. O controle dos passos de carga foi efetuado a partir da opção "Time increment", onde foram admitidos os seguintes valores: Time Step Size = 1,0; Minimum Time Step = 0,1 e Maximum Step Size $=1,0$. Utilizou-se como parâmetro de convergência uma tolerância igual a 0,001 , conforme [17].

Valores de calibração para o modelo numérico: Os valores das propriedades elásticas e plásticas, utilizados na calibração do modelo numérico, estão apresentados nas tabelas 1 a 4 , que se seguem.

Tabela 1. Constantes para o aço dos conectores.

\begin{tabular}{|c|c|c|}
\hline Parâmetro (P) & Valor (V) & Unidade (U) \\
\hline $\mathrm{E}$ & 210000 & $\mathrm{MPa}$ \\
\hline$\sigma_{\mathrm{p}}$ & 500 & $\mathrm{MPa}$ \\
\hline $\mathrm{E}_{\mathrm{T}}$ & 3800 & $\mathrm{MPa}$ \\
\hline$v$ & 0,3 & - \\
\hline$\rho$ & $7,85 \mathrm{E}-05$ & $\mathrm{kN} / \mathrm{cm}^{3}$ \\
\hline
\end{tabular}

Tabela 2. Constantes para o aço das armaduras.

\begin{tabular}{|c|c|c|}
\hline$(\mathrm{P})$ & $(\mathrm{V})$ & $(\mathrm{U})$ \\
\hline $\mathrm{E}$ & 210000 & $\mathrm{MPa}$ \\
\hline$\sigma_{\mathrm{p}}$ & 500 & $\mathrm{MPa}$ \\
\hline $\mathrm{E}_{\mathrm{T}}$ & 210 & $\mathrm{MPa}$ \\
\hline$v$ & 0,3 & - \\
\hline$\rho$ & $7,85 \mathrm{E}-05$ & $\mathrm{kN} / \mathrm{cm}^{3}$ \\
\hline
\end{tabular}

Tabela 3. Constantes para o concreto.

\begin{tabular}{|c|c|c|}
\hline$(\mathrm{P})$ & $(\mathrm{V})$ & $(\mathrm{U})$ \\
\hline $\mathrm{E}$ & 26323,40 & $\mathrm{MPa}$ \\
\hline$\sigma_{\mathrm{p}}$ & 28,11 & $\mathrm{MPa}$ \\
\hline$v$ & 0,2 & - \\
\hline$\rho$ & $2,50 \mathrm{E}-05$ & $\mathrm{kN} / \mathrm{cm}^{3}$ \\
\hline
\end{tabular}


Tabela 4. Constantes para a madeira.

\begin{tabular}{|c|c|c|c|c|c|}
\hline$(\mathrm{P})$ & $(\mathrm{V})$ & $(\mathrm{U})$ & $(\mathrm{P})$ & $(\mathrm{V})$ & $(\mathrm{U})$ \\
\hline $\mathrm{E}_{\mathrm{x}} \pm$ & 1970 & $\mathrm{MPa}$ & $\mathrm{G}_{\mathrm{xy}}$ & 984 & $\mathrm{MPa}$ \\
\hline $\mathrm{E}_{\mathrm{y}} \pm$ & 1970 & $\mathrm{MPa}$ & $\mathrm{G}_{\mathrm{yz}}$ & 984 & $\mathrm{MPa}$ \\
\hline $\mathrm{E}_{\mathrm{z}} \pm$ & 19680 & $\mathrm{MPa}$ & $\mathrm{G}_{\mathrm{xz}}$ & 984 & $\mathrm{MPa}$ \\
\hline$v_{\mathrm{xy}}$ & 0,23 & - & - & - & - \\
\hline$v_{\mathrm{yz}}$ & 0,013 & - & - & - & - \\
\hline$v_{\mathrm{xz}}$ & 0,013 & - & $\rho$ & $1,0 \mathrm{E}-05$ & $\mathrm{kN} / \mathrm{cm}^{3}$ \\
\hline$\sigma_{\mathrm{x}} \pm$ & 11,90 & $\mathrm{MPa}$ & $\mathrm{E}_{\mathrm{Tx}} \pm$ & 4,10 & $\mathrm{MPa}$ \\
\hline$\sigma_{\mathrm{y}} \pm$ & 11,90 & $\mathrm{MPa}$ & $\mathrm{E}_{\mathrm{Ty}} \pm$ & 4,10 & $\mathrm{MPa}$ \\
\hline$\sigma_{\mathrm{z}} \pm$ & 62,80 & $\mathrm{MPa}$ & $\mathrm{E}_{\mathrm{Tz}} \pm$ & 280 & $\mathrm{MPa}$ \\
\hline$\tau_{\mathrm{xy}}$ & 23,90 & $\mathrm{MPa}$ & $\mathrm{G}_{\mathrm{Txy}}$ & 18 & $\mathrm{MPa}$ \\
\hline$\tau_{\mathrm{yz}}$ & 23,90 & $\mathrm{MPa}$ & $\mathrm{G}_{\mathrm{Tyz}}$ & 18 & $\mathrm{MPa}$ \\
\hline$\tau_{\mathrm{xz}}$ & 2,40 & $\mathrm{MPa}$ & $\mathrm{G}_{\mathrm{Txz}}$ & 0,018 & $\mathrm{MPa}$ \\
\hline
\end{tabular}

\section{RESULTADOS E DISCUSSÕES}

Na calibração do modelo a curva "experimental", apresentada foi plotada até o valor último de ruptura, conforme obtido experimentalmente. A curva "numérica" foi plotada até o ponto máximo onde foi possível a obtenção de convergência dos resultados em correspondência a uma tolerância de 0,001 . Portanto, não foi possível a obtenção do comportamento da curva numérica Força versus Deslocamento para valores próximos aos valores de ruptura.

O valores de rigidez $\mathrm{K}$ (Força/deslocamento), para cada conector $\mathrm{X}$, obtido a partir do ensaio experimental do corpo-de-prova misto, foi de $30,26 \mathrm{KN} / \mathrm{mm}$. O valor do coeficiente de rigidez $\mathrm{K}$, obtido através do modelo numérico, foi de $29,17 \mathrm{kN} / \mathrm{mm}$.

$\mathrm{O}$ valor experimental da rigidez $\mathrm{K}$ foi obtido a partir da curva Força versus Deslocamento, com a secante passando pelos pontos $10 \%$ e $40 \%$ da força última na ligação, segundo recomendações [16]. Na verificação da rigidez, a partir da simulação numérica, os deslocamentos referentes aos carregamentos $10 \%$ e $40 \%$ foram tomados no ponto indicado na figura 12 .

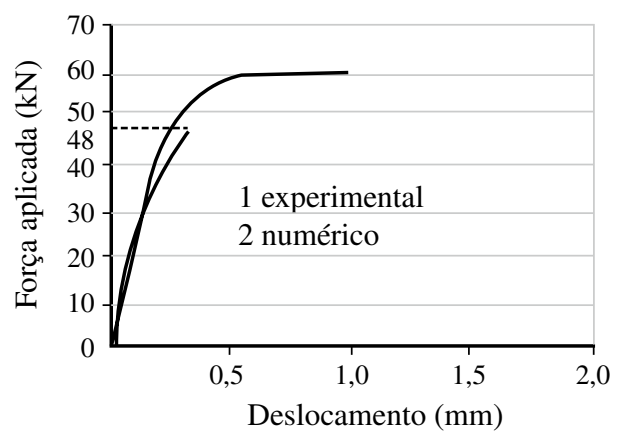

Figura 13. Calibração do modelo para força aplicada de 61,16 kN. Fonte: Adaptado de [8].

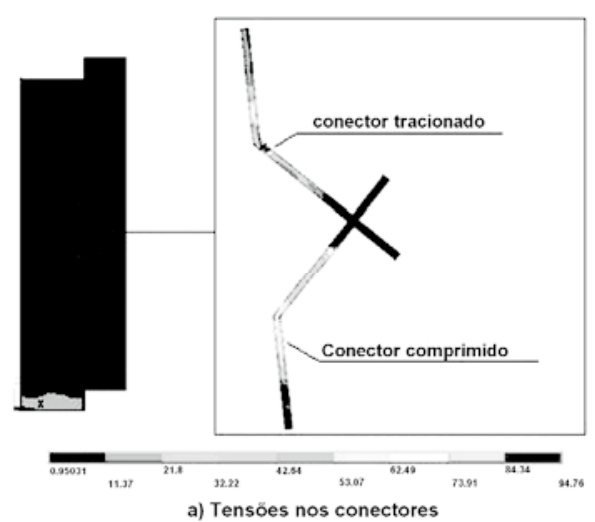

a) Tensöes nos conectores

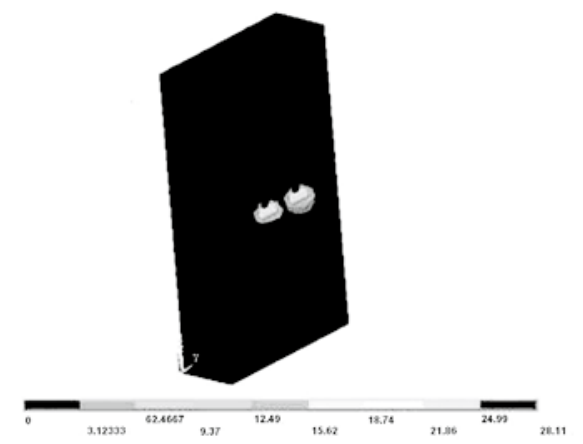

b) Tensōes no concreto

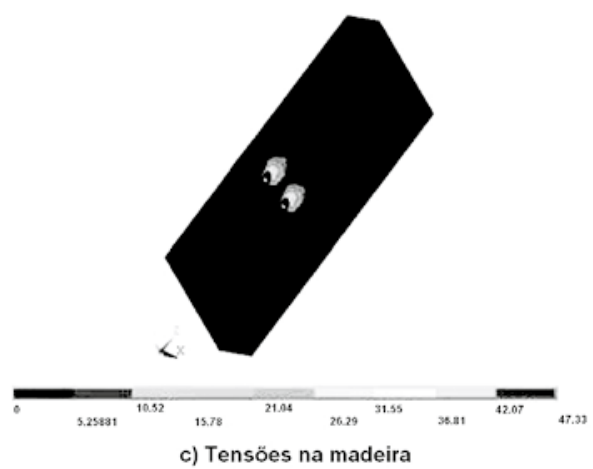

Figura 14. Estado de tensão (MPa) no modelo para força de 61,16kN. Fonte: Adaptado de [8].

A curva de calibração Força versus Deslocamento, obtida para o modelo numérico, tendeu a uma inclinação ascendente, logo após o início do trecho não linear. Esse comportamento, fenômeno conhecido como "travamento numérico", se deve ao fato do elemento finito SOLID45, utilizado na modelagem da peça de madeira, ser um elemento bastante simples e pouco flexível. Neste caso, por mais que se refine a malha dos elementos não se obtém melhoras no comportamento desta curva.

As diferenças observadas entre as curvas numéricas e experimentais podem ser atribuídas a vários fatores: 
Primeiramente, a modelagem da madeira não é uma tarefa fácil devido a possibilidade de existência de imperfeições (distorções nas direções das fibras e presença de nós) que influenciam no seu comportamento, e estas não foram consideradas na simulação numérica. Além disso, a madeira apresenta comportamentos diferentes na tração e na compressão. Na compressão o comportamento da madeira pode ser aproximado por uma lei constitutiva elastoplástica com endurecimento. Na tração, o comportamento da madeira é elasto-frágil e, neste caso, o modelo elastoplástico não representa o comportamento do material.

Das propriedades físicas da madeira a mais importante em termos de simulação numérica foi a tensão de plastificação. Logo que se inicia o processo de carga se inicia também o processo de plastificação e, por esta razão, as propriedades elásticas perdem quase que completamente a influência que exercem no início do comportamento da curva Força versus Deslocamento.

Após a simulação do carregamento, com valor aproximado de $48 \mathrm{kN}$, observou-se que as tensões equivalentes nos materiais madeira e concreto, nas regiões dos conectores, ultrapassaram as resistências médias à compressão, $\mathrm{f}_{\mathrm{co}, \mathrm{m}}$ e $\mathrm{f}_{\mathrm{c}, \mathrm{m}}$, admitidas para os respectivos materiais. Em decorrência desse fato surgiram nestas regiões deformações permanentes que provocaram a perda de rigidez do sistema de conexão.

No modelo numérico, os valores das máximas tensões de compressão, nas regiões dos conectores, foram aproximadamente quatro a cinco vezes maiores que os valores das tensões nas bases de apoio do modelo. Em nenhuma região dos conectores foi atingida a tensão de escoamento do aço. A estratégia de modelagem adotada possibilitou a visualização da concentração de tensões nas regiões de transferência de esforços.

\section{CONCLUSÕES}

O modelo numérico proposto, a partir da utilização do software ANSYS, apresentou correspondência com os resultados experimentais para o carregamento de serviço e foi capaz de simular o comportamento mecânico do sistema misto, não somente na fase elástica linear, mas também no inicio da fase não linear, quando se inicia o processo de plastificação dos materiais. O erro obtido entre as curvas numérica e experimental, neste caso, foi de até $15 \%$.

A partir do modelo proposto não foi possível prever o comportamento da curva Força versus Deslocamento para valores últimos de ruptura, pois os elementos finitos utilizados consideram somente efeitos de elasticidade e plasticidade dos materiais.

A simulação numérica com a representação dos conectores por elementos sólidos (e não por molas ou elementos de barra), possibilitou a análise do comportamento do sistema misto, não somente sobre o aspecto global, a partir da relação Força versus Deslocamento, mas também com relação aos aspectos localizados, como a verificação das tensões nas regiões dos conectores e demais componentes do modelo.

A estratégia de modelagem desenvolvida pode ser utilizada para demais sistemas estruturais, a partir da consideração de outros materiais que apresentem comportamentos isotrópicos e também ortotrópicos, desde que as propriedades elásticas e plásticas dos materiais sejam perfeitamente definidas para cada caso.

\section{AGRADECIMENTOS}

À FAPESP pelo apoio financeiro da pesquisa.

\section{REFERENCIAS}

[1] P.G.A. Segundinho. "Análise teórico-experimental de um tabuleiro misto madeira-concreto composto por vigas circulares". Dissertação (Mestrado). Faculdade de Engenharia de Ilha Solteira. UNESP. Ilha Solteira, SP, Brasil. 2005.

[2] J.L.N. Góes. "Field Performance of a Composite Log-Concrete Bridge". Structural Engineering International. Vol. 18, pp. 79-83. 2008.

[3] J. Soriano. "Estruturas mistas em concreto e madeira: análise de vigas e painéis e aplicações na construção civil". Tese (Doutorado). Faculdade de Engenharia Agrícola. UNICAMP. Campinas, Brasil. 2001.

[4] C.R. de Souza. "Utilização de estruturas mistas na recuperação da capacidade de carga das pontes de madeira do Estado do Mato Grosso do Sul.”. Dissertação (Mestrado). Faculdade de Engenharia de Ilha Solteira. UNESP. Ilha Solteira, SP, Brasil. 2004.

[5] E.S. Flores, C.A. Rioseco, A.V. Matamal. "Calibración del modelo de Hill modificado para el Pino Radiata cileno en conexiones de cizalle doble 
sometidas a compressión paralela”. II Jornadas Chilenas de Estructuras de Madera. Santiago, Chile. 12-15 Noviembre 2007.

[6] A.M.P.G. Días. "Mechanical behavior of timberconcrete joints". Thesis (Doctorate). Universidade de Coimbra. Portugal. 2005.

[7] J.L. Miotto. "Estruturas mistas de madeira-concreto: avaliação de madeira laminada colada com Fibras de vidro". Tese (Doutorado). Escola de Engenharia de São Carlos. Universidade de São Paulo. São Carlos, Brasil. 2009.

[8] J.C. Molina. "Análise do comportamento dinâmico da ligação formada por barras de aço coladas para tabuleiros mistos de madeira e concreto para pontes". Tese (Doutorado). Escola de Engenharia de São Carlos. Universidade de São Paulo. São Carlos, Brasil. 2008.

[9] R. Hill. "A Theory of the Yelding and Plastic Flow of Anisotropic Metals". Proceedings of the Royal Society, Series A, 193, pp. 281-297. 1974.

[10] E.A. Nicolas. "Estudo de critérios de resistência de materiais anisotrópicos aplicados a madeira". Tese (Doutorado). Faculdade de Engenharia Civil. Arquitetura e Urbanismo da Universidade de Campinas. Campinas, Brasil. 2006.
[11] ANSYS INC. “Ansys Release 9.0”. Documentation. 2004.

[12] ASSOCIAÇÃO BRASILEIRA DE NORMAS TÉCNICAS. NBR 7190 - Projeto de Estruturas de Madeira. ABNT. Rio de Janeiro, Brasil. 1997.

[13] TRUEGRID "XYZ Scientific Applications, Inc. version 2.10". Documentation. 2001.

[14] T.I. Kotinda. "Modelagem numérica de vigas mistas aço-concreto simplesmente apoiadas: ênfase ao estudo da interface laje-viga". Dissertação (Mestrado). Escola de Engenharia de São Carlos. Universidade de São Paulo. São Paulo, Brasil. 2004.

[15] A.W. Balarin e M. Nogueira. "Caracterização elástica da Madeira de Eucalipto citriodora". Cerne. Vol. $9 \mathrm{~N}^{\circ}$ 1, pp. 066-080. 2003.

[16] J. C. Pigozzo. "Determinação do módulo de deslizamento e resistência limite de conectores de cisalhamento para estruturas mistas de madeira e concreto". X EBRAMEM. Anais, p. 16. 2006.

[17] J.C. Molina e C.C. Junior. "Análise dinâmica de conectores verticais para tabuleiros mistos de pontes". XI Encontro Brasileiro em Madeiras e Estruturas de Madeira". Londrina, Brasil. Julho 2008. 